\title{
GEOPHYSICS
}

\section{Wave-equation Rayleigh wave dispersion inversion using fundamental and higher modes}

\begin{tabular}{|r|l|}
\hline Journal: & Geophysics \\
\hline Manuscript ID & GEO-2018-0506.R3 \\
\hline Manuscript Type: & Technical Paper \\
\hline Keywords: & surface wave, dispersion, inversion, near surface \\
\hline Area of Expertise: & Engineering and Environmental Geophysics, Seismic Inversion \\
\hline
\end{tabular}

\section{SCHOLARONE ${ }^{m}$ \\ Manuscripts}




\title{
Wave-equation Rayleigh wave dispersion inversion using fundamental and higher modes
}

\author{
Zhen-dong Zhang[1] and Tariq Alkhalifah[1] \\ 1 Department of Physical Science and Engineering, \\ King Abdullah University of Science and Technology, \\ Thuwal 23955-6900, Saudi Arabia.
}

Email: zhendong.zhang@kaust.edu.sa

(February 13, 2019)

Running head: Surface wave inversion 


\begin{abstract}
Recorded surface waves often provide reasonable estimates of the S-wave velocity in the near surface. However, existing algorithms are mainly based on the 1D layered-model assumption and require picking the dispersion curves either automatically or manually. We propose a wave-equation based inversion algorithm that inverts for S-wave velocities using fundamental- and higher-modes Rayleigh waves without picking an explicit dispersion curve. The proposed method aims to maximize the similarity of the phase velocity spectrum $(f-v)$ of the observed and predicted surface waves with all-Rayleigh wave modes (if they exist) included in the inversion. The $f-v$ spectrum is calculated using the linear Radon transform applied to a local similarity-based objective function, and thus, we do not need to pick velocities in spectrum plots. As a result, the best match between the predicted and observed $f-v$ spectrum provides the optimal estimation of the S-wave velocity. We derive S-wave velocity updates using the adjoint-state method and solve the optimization problem using an IBFGS algorithm. Our method excels in cases where the S-wave velocity have vertical reversals and lateral variations, as we utilize all-modes dispersion, and it can suppress the local minimum problem often associated with full waveform inversion applications. Synthetic and field examples are used to verify the effectiveness of the proposed method.
\end{abstract}

Keywords: Rayleigh waves, Dispersion, Higher modes, Inversion. 


\section{INTRODUCTION}

Surface waves are usually considered as a nuisance to the conventional seismic processing of land data. However, such a nuisance actually includes high-quality information for S-wave velocity estimation in the near surface. Based on the complexity of the S-wave velocity variations, Rayleigh wave inversion methods fall into three categories: First, 1D inversion methods, which are usually based on semi-analytical solutions to approximate the elastic wave equation solutions (Nazarian et al., 1983; Xia et al., 2004; Lin et al., 2007; Maraschini et al., 2010; Milana et al., 2014) and stochastic inversion using elastic wave equation solutions (Feng et al., 2005; Dong et al., 2014). Limited by the simplified physics to make the computation affordable, the inversion results are limited to 1D layered models. Second, 2D inversion using the fundamental mode. Among such methods are the wave equation dispersion-curve based inversion (Zhang et al., 2015, 2016; Li et al., 2016; Lu et al., 2017). In practice, the automatically picked dispersion curves are limited to the fundamental mode of Rayleigh waves, and thus, the application of this method is limited to the S-wave velocities with positive gradients in depth. Finally, Elastic full waveform inversion (FWI). Full waveform inversion aims to match the waveform of Rayleigh waves and can invert for complex models (Solano et al., 2013; Groos et al., 2014; Pérez Solano et al., 2014; Yuan et al., 2015; Masoni et al., 2016). However, the method requires good initial models to avoid cycle skipping, which is usually not affordable in practice (Virieux and Operto, 2009; Liu et al., 2018). The 1D inversion scheme can be robust and efficient, but it is less accurate as the lateral variation in the subsurface could be large. In contrast, elastic FWI is less efficient and sensitive to simplified physics used in wavefield extrapolation (Zhang et al., 2014), but it is able to invert for complex models. Among all these methods, the wave-equation dispersion inversion method is a compromise between the $1 \mathrm{D}$ inversion scheme and the waveform 
inversion.

Instead of fitting complex waveforms, the wave-equation dispersion inversion method (also known as skeletonized inversion) aims to match the frequency-dependent phase velocities of Rayleigh waves. It has a more quasi-linear relationship between the S-wave velocity and the dispersion curve and has a less bumpy misfit function than that corresponding to waveform inversion (Zhang et al., 2016). However, the previously proposed wave-equation inversion algorithms highly depend on the automatic picking of the dispersion curves from the $f-v$ spectrum. Although automatic picking is often applied to the predicted data, in which case the approach is stable, it inevitably ignores the higher-mode Rayleigh waves and therefore, cannot handle models with low-velocity layers below high-velocity ones (velocity reversal). The generation of higher modes is often attributed to the presence of low S-wave velocity layers (STOKOE II, 1994), and thus, such low velocity (or velocity reversal) cannot be recovered without inverting such modes. Besides, high-mode Rayleigh waves penetrate deeper than its fundamental mode and can increase the resolution of the estimated S-wave velocities (Xia et al., 2003; Beaty and Schmitt, 2003; Luo et al., 2007).

In this paper, we adopt a wave equation dispersion inversion method to include the fundamental- and higher-modes Rayleigh waves. $f-v$ spectra instead of the dispersion curves are used as input data, which avoids the picking process. A local-similarity based objective function is introduced to measure the similarity of the observed and predicted $f-v$ spectra. The $f-v$ spectrum is calculated using the linear Radon transform (Luo et al., 2008). This paper has five sections. After the introduction, we introduce a novel objective function and solve the optimization problem. In the examples, we first illustrate the synthetic case with S-wave velocity reversal and lateral variation, then apply the method to the field data set collected across the Qademah faults in Saudi Arabia. The proposed 
method applies to more general inverse problems and also has effectiveness and limitations as discussed in the discussion. At last, we summarize our work and draw conclusions.

\section{THEORY}

The basic concept of the wave-equation dispersion curve inversion is illustrated in Figure 1 (Zhang et al., 2016). Specifically, Figure 1a shows a number of Rayleigh-wave cycles in traces and so the waveform-fitting measurement for such data is expected to be cycleskipped when the initial model is far from the actual one. Therefore, we should look for simpler data representations, which preserve the vital attributes but have fewer cycles. One such reduction is to use the frequency-dependent phase velocities, $C(\omega)=\omega / k(\omega)$, for the fundamental-mode Rayleigh waves as shown in Figure $1 \mathrm{~b}$ to estimate S-wave velocities. The explicit dispersion curve is identified by the maximum amplitudes with the closet proximity to the wavenumber axis in the $\omega-k$ spectrum as shown in Figure 1c. However, automatically picking such a stationary curve is not easy for noisy data and current automatic picking methods ignore the weaker higher-order dispersion modes. Utilizing dispersion spectra under the framework of FWI can mimic the goal of using all dispersion modes without picking. However, we need to find a better inversion scheme that can suppress cycle skipping often faced by conventional FWI.

Objective functions for FWI intend to measure the differences in the observed and predicted data. One of the most intuitive mismatch measurements is the $L_{2}$ norm distance, which is given by

$$
\phi(m)=\left\|d^{p}(m)-d^{o}(m)\right\|^{2}
$$

where $d^{p}(m)$ and $d^{o}(m)$ are predicted and observed data, respectively. 
The inverse problem is constrained by the elastic wave equation given by

$$
\left(\begin{array}{cc}
\rho \mathbf{I}_{3} & 0 \\
0 & \mathbf{C}^{-1}
\end{array}\right) \frac{\partial \Psi}{\partial t}-\left(\begin{array}{cc}
0 & E^{T} \\
E & 0
\end{array}\right) \Psi-\mathbf{s}=0
$$

where $\Psi=\left(v_{1}, v_{2}, v_{3}, \sigma_{1}, \sigma_{2}, \sigma_{3}, \sigma_{4}, \sigma_{5}, \sigma_{6}\right)^{T}$ is the vector containing three particle velocities and six stresses in different directions as noted by the numbers, $E$ denotes spatialdifferentiation operators, $\mathbf{I}_{3}$ is a 3 by 3 identity matrix. $\mathbf{C}$ represents the stiffness matrix and $\mathbf{s}$ denotes the point source used for modeling.

Due to the oscillatory nature of seismic waves, the $L_{2}$ norm objective function suffers from cycle skipping when the mismatches between the predicted and observed data exceed a half cycle. A natural remedy to this problem is to compare two events within a predefined extension (Fomel, 2007). We propose a local-similarity based objective function replacing the $L_{2}$ norm based objective function, which is given by

$$
\phi(m)=\frac{1}{2} \int_{s} \int_{r} \int_{f} \int_{f^{\prime}} W\left(f^{\prime}\right) C_{w}^{2}\left(f, v, f^{\prime}\right) d f^{\prime} d f d r d s
$$

where $C_{w}^{2}\left(f, v, f^{\prime}\right)$ denotes the local-crosscorrelation of predicted and observed $f-v$ spectra. $f^{\prime}$ denotes frequency extensions. $s$ and $r$ are source and receivers, respectively. $W\left(f^{\prime}\right)$ is a polynomial-type weighting function, which satisfies the following boundary conditions: $\left.W\right|_{ \pm f^{\prime}}=0 ;\left.W^{\prime}\right|_{ \pm f^{\prime}}=0 ;\left.W\right|_{0}=1 ;\left.W^{\prime}\right|_{0}=0$. Compared with the widely used linear or Gaussian penalties, the proposed penalty function only needs the maximum extension $\left(f^{\prime}\right)$ as an input parameter and thus is much easier to use (Zhang et al., 2018).

The local-crosscorrelation admits frequency-dependent similarities instead of a single value often admitted by the global-crosscorrelation (Zhang and Alkhalifah, 2018). The 
definition of local-crosscorrelation is given by

$$
C_{w}\left(f, v, f^{\prime}\right)=\frac{\int_{f-\frac{1}{2} w}^{f+\frac{1}{2} w}\left|C^{p}(f, v) \| C^{o}\left(f, v, f^{\prime} f\right)\right| d f}{\sqrt{\int_{f-\frac{1}{2} w}^{f+\frac{1}{2} w}\left|C^{p}(f, v)\right|^{2} d f} \sqrt{\int_{f-\frac{1}{2} w}^{f+\frac{1}{2} w}\left|C^{o}\left(f, v, f^{\prime} f\right)\right|^{2} d f}},
$$

where $\left|C^{p}(f, v)\right|$ and $\left|C^{o}\left(f, v, f^{\prime} f\right)\right|$ are the modeled and extended observed spectra, respectively, and $w$ is the length of the window. In practice, the local-similarity is calculated by solving a least-squares problem with shaping regularizations (Fomel, 2007):

$$
\mathbf{c}_{w}=\frac{\mathbf{S U}^{T} \mathbf{m}}{\lambda^{2} \mathbf{I}+\mathbf{S}\left(\mathbf{U}^{T} \mathbf{U}-\lambda^{2} \mathbf{I}\right)} \odot \frac{\mathbf{S M}^{T} \mathbf{u}}{\lambda^{2} \mathbf{I}+\mathbf{S}\left(\mathbf{M}^{T} \mathbf{M}-\lambda^{2} \mathbf{I}\right)} .
$$

Thus, the local-similarity attributes are calculated by a product of two least-squares inverses using shaping regularization. The $\mathbf{S}$ in equation 5 denotes the shaping operator, and $\mathbf{U}$ and $\mathbf{M}$ are diagonal matrices, which are composed of $\left|C^{p}(f, v)\right|$ and $\left|C^{o}\left(f, v, f^{\prime} f\right)\right|$, respectively. I is the identity matrix and $\lambda$ is the damping factor, and $\odot$ denotes the component-wise product.

The $f-v$ spectrum is calculated using a linear Radon transform (Luo et al., 2008). After a temporal Fourier transform of the shot gather, the linear Radon transform can be calculated for each temporal frequency component $f$ as:

$$
C(f, v)=\int_{x_{\min }}^{x_{\max }} D(f, x) e^{-\frac{i 2 \pi f x}{v}} d x
$$

and its adjoint form is given by

$$
D(f, x)=\int_{v_{\min }}^{v_{\max }} C(f, v) e^{\frac{i 2 \pi f x}{v}} d v
$$

The gradient for S-wave velocity updating with respect to the objective function can be calculated by the chain rule, which is $\frac{\partial \phi(m)}{\partial m}=\frac{\partial \phi(m)}{\partial d^{p}(m)} \frac{\partial d^{p}(m)}{\partial m}$, where $\frac{\partial \phi(m)}{\partial d^{p}(m)}$ is the adjoint source and $\frac{\partial d^{p}(m)}{\partial m}$ is the Fréchet derivative (Plessix, 2006). The adjoint source of the 
proposed objective function would be (Solano et al., 2013; Zhang et al., 2018)

$$
\frac{\partial \phi(m)}{\partial d^{p}(m)}=\Re\left(i F F T\left(\operatorname{AdjRadon}\left(\frac{\partial \phi(m)}{\partial\left|C^{p}\right|} \frac{\partial\left|C^{p}\right|}{\partial C^{p}}\right)\right)\right)
$$

and

$$
\begin{gathered}
\frac{\partial \phi(m)}{\partial\left|C^{p}\right|}= \\
\int_{f^{\prime}} W\left(f^{\prime}\right) C_{w}\left(f, v, f^{\prime}\right) \\
\left(\frac{\left|C^{o}\left(f, v, f^{\prime} f\right)\right|}{\sqrt{\int_{f-\frac{1}{2} w}^{f+\frac{1}{2} w}\left|C^{p}(f, v)\right|^{2} d f} \sqrt{\int_{f-\frac{1}{2} w}^{f+\frac{1}{2} w}\left|C^{o}\left(f, v, f^{\prime} f\right)\right|^{2} d f}}-\frac{\left|C^{p}(f, v)\right| \int_{f-\frac{1}{2} w}^{f+\frac{1}{2} w}\left|C^{p}(f, v)\right|\left|C^{o}\left(f, v, f^{\prime} f\right)\right| d f}{\sqrt{\int_{f-\frac{1}{2} w}^{f+\frac{1}{2} w}\left|C^{p}(f, v)\right|^{2} d t} \sqrt[3]{\int_{f-\frac{1}{2} w}^{f+\frac{1}{2} w}\left|C^{o}\left(f, v, f^{\prime} f\right)\right| d f}}\right) d f^{\prime} .
\end{gathered}
$$

By cross correlating of the forward-propagated source wavefield and the backwardpropagated adjoint-source (equations 8 and 9) wavefield, we can obtain the gradient of the objective function. S-wave velocity is iteratively updated using the Limited-memory Broyden-Fletcher-Goldfarb-Shanno (lBFGS) optimization scheme (Liu and Nocedal, 1989), which can be written as

$$
m=m_{0}-\alpha H^{-1} g
$$

where $H^{-1}$ denotes the inverse Hessian which is approximated by the finite-difference scheme in IBFGS and $\alpha$ is the step length used for S-wave velocity updates.

In summary, the proposed inversion method has three main steps:

1. Fourier transform of common shot gathers in time.

2. Calculate the corresponding $f-v$ spectrum (equation 6 ).

3. Solve the optimization problem using equations 3 and 10.

The implementation of the dispersion spectrum inversion is straightforward. The linearradon transform enhances the signal-to-noise ratio (SNR) of the surface waves, and thus, 
only a few preprocessing steps are needed. The benefits of the approach are: 1) No need to pick dispersion curves; 2) More wave modes (if they exist) are included in the inversion, which can handle velocity reversals and provide a better estimation of the subsurface.

\section{NUMERICAL EXAMPLES}

We use both synthetic and field data examples to test our proposed method. The P-wave velocity and density used for synthetic examples are layered models as shown in Figures $2 \mathrm{a}$ and $2 \mathrm{~b}$, respectively. We use the true density model for inversion, but the $\mathrm{P}$-wave velocity used for the inversion is $10 \%$ lower or higher than its actual value in the synthetic case. The input P-wave velocity used for field data is a linearly increasing one. Initial S-wave velocities are both linearly increasing in the synthetic and field examples. Its lower and upper boundaries are determined by the range of phase velocities in the dispersion spectrum. The surface waves are simulated by solving the elastic wave equation (equation 2) with a free-surface boundary condition.

\section{Synthetic S-wave velocity with negative gradient in depth}

We first test our method on a layered model with low-velocity layers below high-velocity ones (velocity reversals). There are 196 vertical sources and 196 receivers (vertical- and horizontal-components) distributed on the top surface with a constant increment. The maximum frequency used in the inversion is $30 \mathrm{~Hz}$ and the spatial sampling is $4 \mathrm{~m}$. The actual S-wave velocity as shown in Figure 3a is a layered one with velocity reversals, in which case the fundamental-mode based Rayleigh wave inversion, as expected, fails to recover the low-velocity layers. The linear gradient initial velocity as shown in Figure $3 \mathrm{~b}$ is determined 
by the observed dispersion spectrum shown in Figure 3k. We conduct three experiments which are 1) using the actual P-wave velocity, 2) a $10 \%$ lower one and 3) a $10 \%$ higher one as input $\mathrm{P}$-wave velocities. The inverted $\mathrm{S}$-wave velocities are shown in Figures $3 \mathrm{c}, 3 \mathrm{~d}$ and 3e, respectively. The horizontal-component common-shot-gathers (CSG) comparison (Figures $3 \mathrm{f} \sim 3 \mathrm{j}$ ) indicates that the data are matching with each other although our proposed objective aims to match the dispersion spectrum. This reasonably indicates that there is a unique solution by either matching the waveforms or the dispersion spectrum. The inverted dispersion spectra (Figures $3 \mathrm{~m} \sim 3 \mathrm{o}$ ) are closer to the observed one (Figure 3k) compared with the initial one (Figure 31). We then choose vertical profiles in the middle of the models for a better comparison as shown in Figure 4. Inverted S-wave velocities are similar to each other even with different input P-wave velocities. Both of them are close to the actual S-wave velocity. There is an apparent difference in the deeper part, which might be caused by the insufficient S-wave illumination.

\section{Synthetic S-wave velocity with lateral variations}

Considering practical applications, the proposed method should be able to invert lateral inhomogeneities. We use a checkerboard model to verify its effectiveness. The actual S-wave velocity in Figure 5a has both lateral variations and depth reversals. The same observation geometry and parameters are used as in the first example. We use a linear-gradient initial S-wave velocity (Figure 5b) and an inaccurate P-wave velocity (90\%) to obtain the updated model shown in Figure 5c. CSGs (Figure 5d 5f) and their corresponding dispersion spectra (Figure $5 \mathrm{~g} \sim 5 \mathrm{i}$ ) indicate that the proposed inversion can match the observed dispersion spectrum and also its corresponding CSGs. We then use a constant initial model (Figure 6a) to further verify the effectiveness of the proposed method. We compare the conventional 
$L_{2}$ norm elastic FWI (waveform subtraction) with the proposed method as shown in Figures $6 \mathrm{~b}$ and $6 \mathrm{c}$, respectively. The predicted data (Figure $6 \mathrm{~d}$ ) and its dispersion spectrum (Figure $6 \mathrm{~g}$ ) indicate that the constant initial model is far from the actual model. Conventional $L_{2}$ norm based inversion fails to match the observed data (as shown in Figure 6e) so does the dispersion spectrum indicate (Figure 6h). However, both the CSGs and the dispersion spectrum (Figures 6f and 6i) from the proposed inversion are closer to the observed ones than the conventional $L_{2}$ norm based inversion. The proposed inversion is not perfect due to the insufficient illumination at the deep part and also considering the acquisition boundaries. To further improve the inverted velocity, we add a total variation (TV) constraint to the inverted model as suggested by Guitton (2012) and Alkhalifah et al. (2018). Figure 7a shows the improved estimation which has a much better recovery for the deep layer. Both the CSGs and its dispersion spectrum (Figures $7 \mathrm{~b}$ and $7 \mathrm{c}$ ) indicate a good match with the observed ones. At last, we use the improved model (Figure 7a) as the initial model for a conventional $L_{2}$ norm elastic FWI. Both the predicted data from the final inverted model and the dispersion spectrum (Figures $7 \mathrm{e}$ and $7 \mathrm{f}$ ) indicate that the model obtained from the proposed method plus the TV constraint can be a good initial model for conventional elastic FWI. For the experiment with the constant initial model, we use the actual P-wave velocity as input in order to allow us to conduct the conventional elastic FWI, which is more sensitive to the P-wave velocity than the proposed approach. Figure 8 shows three vertical profiles at different lateral locations (yellow triangles in Figure 5a). We can find that by either using a better initial model (linear gradient) or using a bad initial one (constant), but with TV constraints; our proposed method can obtain similar inversion results. 


\section{Field data example}

A seismic land survey (blue line marked P-2 in Figure 9) was acquired across the Qademah fault near the Red Sea coast in Saudi Arabia, and we show a representative shot gather in Figure 10a. It is preprocessed by adding a soft-muting window to the raw data set and applying a bandpass filter. Besides, a time-squared gain is applied as suggested by Claerbout (1985) as shown in Figure 10b. 120 receivers (vertical component) and 120 vertical sources with a sampling of 5 meters are deployed evenly on the surface. The dominant frequency of the raw data is $40 \mathrm{~Hz}$. We filtered the data to a maximum frequency of $30 \mathrm{~Hz}$ for an inversion purpose. A Ricker wavelet with a frequency band of 10-20 Hz (inline with the frequency components of the observed data) is used as the source wavelet. The $f-v$ spectra calculated using equation 6 for the raw and preprocessed shots are shown in Figures 10c and $10 \mathrm{~d}$, respectively. It's clear that the dispersion spectrum is improved after proper data preprocessing. The pink contour curves indicate the dominant modes and it is used for later comparison. The initial $\mathrm{P}$-wave velocity is a linear gradient one, which is determined by the P-wave tomography model for this area and the initial density model is calculated using Gardner's equation. The initial S-wave velocity (Figure 11a) is a linearly increasing model and the observed $f-v$ spectrum roughly decides its minimum and maximum values. The estimated S-wave velocity is plotted in Figure 11b. There are some low-velocity zones as expected since the line is across the fault area and these low-velocity zones cannot be recovered using only fundamental-mode dispersion. As a quality control, we also plot the predicted data from the initial and inverted S-wave velocities and their $f-v$ spectra in Figures 12 (a, c) and $12(\mathrm{~b}, \mathrm{~d})$, respectively. The dispersion spectrum of the proposed inversion can get improved as the pink contour curve indicates. However, the predicted CSGs are not expected to exactly match the observed ones. For reference, the reader can 
compare the results to those attained by Li et al. (2016) of the same line, where they picked the fundamental mode and derived the gradient use the connective function. Our inverted result tends to have a higher resolution, especially in the shallow area, which is expected by utilizing the higher modes (Xia et al., 2004). Besides, we do not need to pick the dispersion mode and the implementation can be easily done by modifying the objective function and the corresponding adjoint-source of the conventional elastic full waveform inversion code.

\section{DISCUSSIONS}

The proposed inversion algorithm can solve general inverse problems. It is also applicable to global-size problems, where the multi-channel seismic data are replaced by the often sparsely placed seismograms. Considering the difference in the coverage used for regional and global seismology, the calculation of dispersion spectrum might be different. The 3D application of the proposed method is also possible. We might need to treat the 3D data volume as several 2D slices and calculate the dispersion spectrum using existing approaches such as the linear Radon transform. It is also possible to obtain a 3D dispersion spectrum and use the same inversion strategy in this paper, which will require more investigation. The proposed method is not limited to Rayleigh waves dispersion inversion. It also applies to other dispersive waves, which have a liner moveout. For example, Love waves also could be used in the proposed inversion (Dokter et al., 2017). The proposed method also has the potential to estimate the anisotropy of the near-surface using the difference in dispersion spectra in the inline and crossline directions.

The applications to synthetic and real data highlight the advantages and limitations of the method. The proposed method can use poor initial models for inversion. Total-variation constraints help recover full-wavenumber components which are usually unavailable with 
band-limited seismic data and space-limited acquisitions (Guitton, 2012; Alkhalifah et al., 2018). The linear stacking can suppress some random noise in the field data, but it can also lower the lateral resolution. The offset range used for stacking can change the lateral resolution. The optimal stacking range might depend on the data and the target area. Also, there are other techniques available to improve the resolution of the $f-v$ spectrum (Zheng and $\mathrm{Hu}, 2017)$. The computational cost of the proposed method can be equivalent to the regular elastic FWI using a single frequency band.

\section{CONCLUSIONS}

We present a multidimensional S-wave velocity estimation method associated with Rayleigh waves based on the elastic wave equation. The main benefits of the proposed method are that it includes the fundamental- and higher-modes (if they exist), we do not need to pick dispersion curves and it suppresses cycle-skipping problems associated with FWI of complex surface waves. Higher modes in the $f-v$ spectrum help increase the penetration depth and resolution in estimating the model and they're necessary for inverting for Swave low-velocity layers. The proposed method is insensitive to random noise and P-waves in the observed data since the linear Radon transform enhances the surface waves with linear moveout. Inaccurate $\mathrm{P}$-wave velocities have limited influence on the S-wave velocity estimation. S-wave velocity updates can be derived from the adjoint-state method and the inverse problem is efficiently solved using the lBFGS optimization method. Both synthetic and field data examples verify the effectiveness of the proposed method and also reveal some of its limitations. One possible limitation of the approach is the reduced lateral resolution after stacking. On the other hand, the lateral resolution can be improved by using multiple shot recordings. 


\section{ACKNOWLEDGMENTS}

We thank Jerry Schuster, Jing Li and Sherif Hanafy for providing the field data and helpful discussions. We want to thank Erika Gasperikova, Antoine Guitton and four anonymous reviewer, for the effort put into the review of this manuscript. We thank KAUST for its support and specifically the seismic wave analysis group members for their valuable insights. For computer time, this research used the resources of the Supercomputing Laboratory at King Abdullah University of Science \& Technology (KAUST) in Thuwal, Saudi Arabia. 


\section{REFERENCES}

Alkhalifah, T., B. B. Sun, and Z. Wu, 2018, Full model wavenumber inversion: Identifying sources of information for the elusive middle model wavenumbers: Geophysics, 83, R597R610.

Beaty, K. S., and D. R. Schmitt, 2003, Repeatability of multimode Rayleigh-wave dispersion studies: Geophysics, 68, 782-790.

Claerbout, J. F., 1985, Imaging the earth's interior: Blackwell scientific publications Oxford, 1.

Dokter, E., D. Köhn, D. Wilken, D. De Nil, and W. Rabbel, 2017, Full waveform inversion of SH-and Love-wave data in near-surface prospecting: Geophysical Prospecting, 65, $216-236$.

Dong, Z., C. Xiaofei, and M. Xiaogui, 2014, Rayleigh wave analysis and inversion for near surface shear wave velocity model building: Beijing 2014 International Geophysical Conference \& Exposition, Beijing, China, 21-24 April 2014, Society of Exploration Geophysicists and Chinese Petroleum Society, 1217-1220.

Feng, S., T. Sugiyama, and H. Yamanaka, 2005, Effectiveness of multi-mode surface wave inversion in shallow engineering site investigations: Exploration Geophysics, 36, 26-33.

Fomel, S., 2007, Local seismic attributes: Geophysics, 72, A29-A33.

Groos, L., M. Schäfer, T. Forbriger, and T. Bohlen, 2014, The role of attenuation in 2D full-waveform inversion of shallow-seismic body and Rayleigh waves: Geophysics, 79, R247-R261.

Guitton, A., 2012, Blocky regularization schemes for full-waveform inversion: Geophysical Prospecting, 60, 870-884.

Hanafy, S. M., A. AlTheyab, and G. T. Schuster, 2015, Controlled noise seismology, in 
SEG Technical Program Expanded Abstracts 2015: Society of Exploration Geophysicists, $5102-5106$.

Li, J., Z. Feng, and G. Schuster, 2016, Wave-equation dispersion inversion: Geophysical Journal International, 208, 1567-1578.

Lin, F.-C., M. H. Ritzwoller, J. Townend, S. Bannister, and M. K. Savage, 2007, Ambient noise Rayleigh wave tomography of New Zealand: Geophysical Journal International, 170, 649-666.

Liu, D. C., and J. Nocedal, 1989, On the limited memory BFGS method for large scale optimization: Mathematical programming, 45, 503-528.

Liu, Y., B. He, H. Lu, Z. Zhang, X.-B. Xie, and Y. Zheng, 2018, Full-intensity waveform inversion: Geophysics, 83, R649-R658.

Lu, K., J. Li, B. Guo, L. Fu, and G. Schuster, 2017, Tutorial for wave-equation inversion of skeletonized data: Interpretation, 5, SO1-SO10.

Luo, Y., J. Xia, J. Liu, Q. Liu, and S. Xu, 2007, Joint inversion of high-frequency surface waves with fundamental and higher modes: Journal of Applied Geophysics, 62, 375-384.

Luo, Y., J. Xia, R. D. Miller, Y. Xu, J. Liu, and Q. Liu, 2008, Rayleigh-wave dispersive energy imaging using a high-resolution linear Radon transform: Pure and Applied Geophysics, 165, 903-922.

Maraschini, M., F. Ernst, S. Foti, and L. V. Socco, 2010, A new misfit function for multimodal inversion of surface waves: Geophysics, 75, G31-G43.

Masoni, I., J.-L. Boelle, R. Brossier, and J. Virieux, 2016, Layer stripping FWI for surface waves, in SEG Technical Program Expanded Abstracts 2016: Society of Exploration Geophysicists, 1369-1373.

Milana, G., P. Bordoni, F. Cara, G. Di Giulio, S. Hailemikael, and A. Rovelli, 2014, 1D 
velocity structure of the Po River plain (Northern Italy) assessed by combining strong motion and ambient noise data: Bulletin of Earthquake engineering, 12, 2195-2209.

Nazarian, S., I. Stokoe, H. Kenneth, and W. Hudson, 1983, Use of spectral analysis of surface waves method for determination of moduli and thicknesses of pavement systems: Transportation Research Board, 38-45.

Pérez Solano, C., D. Donno, and H. Chauris, 2014, Alternative waveform inversion for surface wave analysis in 2-D media: Geophysical Journal International, 198, 1359-1372.

Plessix, R.-E., 2006, A review of the adjoint-state method for computing the gradient of a functional with geophysical applications: Geophysical Journal International, 167, 495503.

Solano, C. P., D. Donno, and H. Chauris, 2013, 2D surface wave inversion in the FK domain: Presented at the 75th EAGE Conference \& Exhibition incorporating SPE EUROPEC 2013.

STOKOE II, K., 1994, Characterization of geotechnical sites by SASW method, in geophysical characterization of sites: ISSMFE Technical Committee\# 10.

Virieux, J., and S. Operto, 2009, An overview of full-waveform inversion in exploration geophysics: Geophysics, 74, WCC1-WCC26.

Xia, J., R. D. Miller, C. B. Park, J. Ivanov, G. Tian, and C. Chen, 2004, Utilization of highfrequency rayleigh waves in near-surface geophysics: The Leading Edge, 23, 753-759.

Xia, J., R. D. Miller, C. B. Park, and G. Tian, 2003, Inversion of high frequency surface waves with fundamental and higher modes: Journal of Applied Geophysics, 52, 45-57.

Yuan, Y. O., F. J. Simons, and E. Bozdă̆, 2015, Multiscale adjoint waveform tomography for surface and body waves: Geophysics, 80, R281-R302.

Zhang, H., M. Maceira, P. Roux, and C. Thurber, 2014, Joint inversion of body-wave 
arrival times and surface-wave dispersion for three-dimensional seismic structure around SAFOD: Pure and Applied Geophysics, 171, 3013-3022.

Zhang, Z., T. Alkhalifah, Z. Wu, Y. Liu, B. He, and J. Oh, 2018, Normalized nonzero-lag crosscorrelation elastic full-waveform inversion: Geophysics, 84, R15-R24.

Zhang, Z.-d., and T. Alkhalifah, 2018, Adaptive data-selection elastic full-waveform inversion, in SEG Technical Program Expanded Abstracts 2018: Society of Exploration Geophysicists, 5163-5167.

Zhang, Z.-d., Y. Liu, and G. Schuster, 2015, Wave equation inversion of skeletonized surfacewaves, in SEG Technical Program Expanded Abstracts 2015: Society of Exploration Geophysicists, 2391-2395.

Zhang, Z.-d., G. Schuster, Y. Liu, S. M. Hanafy, and J. Li, 2016, Wave equation dispersion inversion using a difference approximation to the dispersion-curve misfit gradient: Journal of Applied Geophysics, 133, 9-15.

Zheng, Y., and H. Hu, 2017, Nonlinear signal comparison and high-resolution measurement of surface-wave dispersion: Bulletin of the Seismological Society of America, 107, 15511556. 


\section{LIST OF FIGURES}

1 An example common shot gather is shown in a), whereas b) shows the actual $C(\omega)-\omega$ spectrum of the CSG and c) shows the automatically picked frequency-dependent phase velocity $(C(\omega)=\omega / k(\omega))$ curve from the spectrum (revised after Zhang et al. (2016)).

2 The actual $v_{p}$ a) and density b) models used for the synthetic examples. They're also used as initial models but the initial $v_{p}$ is equal to $90 \%$ of the true model in synthetic examples.

3 A layered model example. a) Actual S-wave velocity with low-velocity layers below high-velocity ones, b) linear gradient initial model. c), d) and e) are recovered S-wave velocities with $100 \%, 90 \%$ and $110 \%$ of the actual P-wave velocity used in the inversion, respectively. f) $\sim \mathrm{j}$ ) are simulated data (horizontal component) using the corresponding $\mathrm{S}$-wave velocities in a) $\sim \mathrm{e}$ ) and their dispersion spectra are shown in $\mathrm{k}$ ) $\sim 0$ ), respectively.

4 The vertical profile in the middle of the model. The low S-wave velocity zones are captured. The inaccurate $\mathrm{P}$-wave velocities have little influence on the inverted S-wave velocity.

5 The checkerboard example with a linear gradient initial model. a) Actual checkerboard model, b) linear gradient initial and c) estimated S-wave velocity. d) f) are simulated shot gathers using the S-wave velocities in a) $\sim$ c) and g) $\sim$ i) are corresponding dispersion spectra. The inverted dispersion spectrum is close to the observed one.

6 The checkerboard example with a constant initial model. a) The constant S-wave velocity model used for inversion, b) estimated S-wave velocity from conventional elastic FWI and c) the one from our proposed method. The simulated shot gathers are shown in 
d) $\sim f$ ) and dispersion spectra of the initial and proposed estimation are shown in $g$ ) and i), respectively. h) shows dispersion spectrum obtained from the $L_{2}$ inversion. The dispersion spectrum of the proposed method is closer to the actual one than the one obtained from $L_{2}$ inversion.

7 The improved inversion by adding TV constraints to the proposed inversion (Figure 6c). a) $\sim$ c) are proposed inversion plus TV constraint. Quality of the deep layer is improved. d) $\sim$ f) are conventional elastic FWI results with a) as input model. It indicates that the proposed method plus TV constraint can provide a good initial model for conventional FWI.

8 Vertical profiles at different lateral locations (yellow triangles in Figure 5). There are two pairs of velocities which are 1) linear gradient initial and the proposed inversion and 2) constant initial and $L_{2}$ norm elastic FWI using the model of proposed inversion plus TV constraints as initial.

9 The seismic survey line across the fault area (Hanafy et al., 2015).

10 Common shot gathers and their dispersion spectra. a) One example raw shot gather and b) preprocessed one. c) and d) are dispersion spectra from a) and b), respectively. The pink contour isolate the dominant modes of Rayleigh waves.

11 The initial S-wave velocity a) and the estimated one b). The initial S-wave velocity can be roughly determined using the range of velocities in the $f-v$ spectrum (Figure 10d).

12 Data comparison. a) and b) are simulated shot gather using the initial and inverted velocities. c) and d) are the corresponding dispersion spectra. The pink contour indicates the area of dominant modes existing in the observed data. 
a) Common Shot Gathers b)
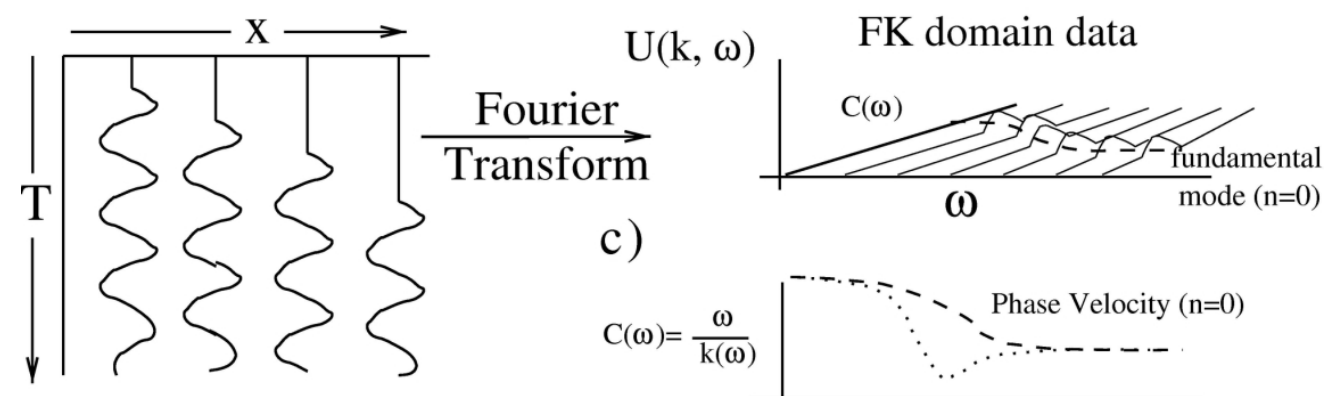

c)

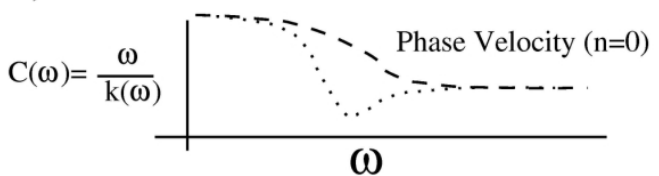

Figure 1 An example common shot gather is shown in $a)$, whereas b) shows the actual $C(\omega)$ - $\omega$ spectrum of the CSG and c) shows the automatically picked frequency-dependent phase velocity $(C(\omega)=\omega / k(\omega))$ curve from the spectrum (revised after Zhang et al., 2016).

$169 \times 65 \mathrm{~mm}(300 \times 300 \mathrm{DPI})$ 


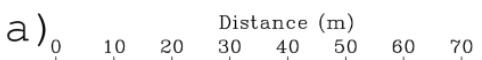

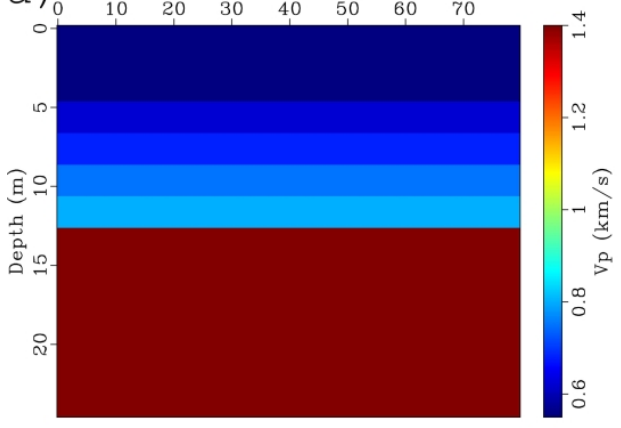

b) $0 \quad 10 \quad 20 \quad$ Distance $(\mathrm{m})$

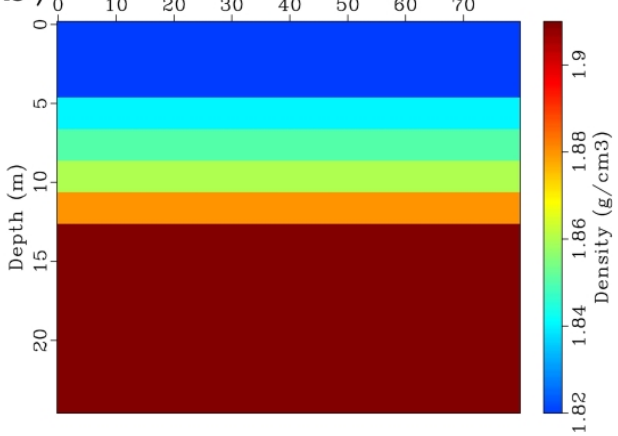

Figure 2 The actual $V p a$ ) and density $b$ ) models used for the synthetic examples. They're also used as initial models but the initial $\mathrm{Vp}$ is equal to $90 \%$ of the true model in synthetic examples.

$324 \times 114 \mathrm{~mm}(300 \times 300$ DPI $)$ 

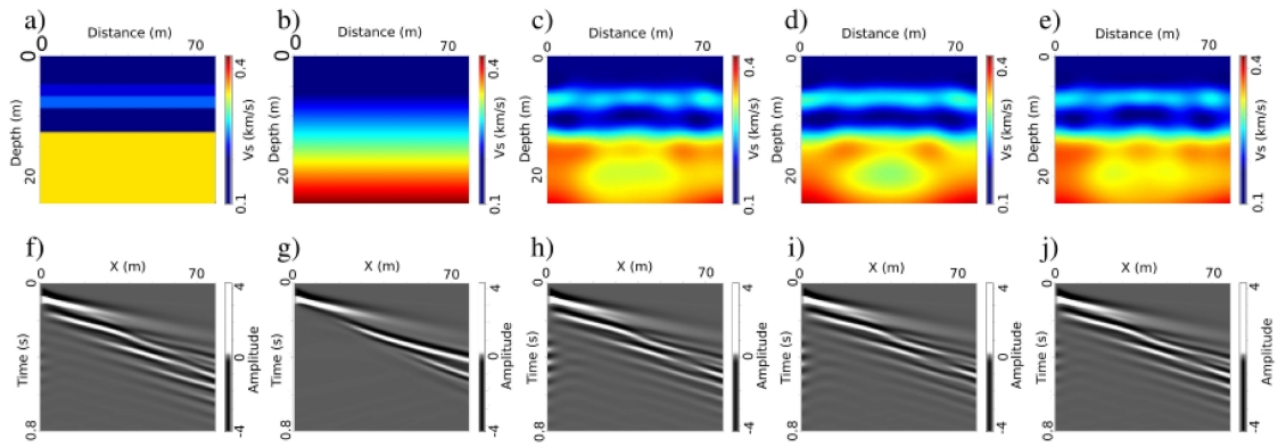

g)

h)
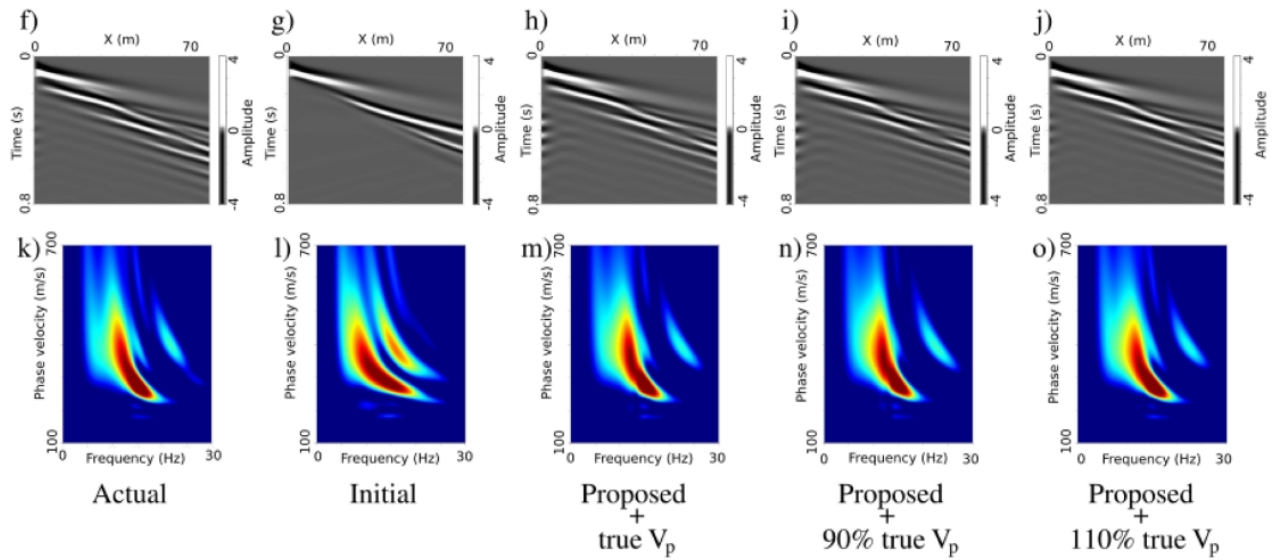

Figure 3 A layered model example. a) Actual S-wave velocity with low-velocity layers below high-velocity ones, b) linear gradient initial model. c), d) and e) are recovered S-wave velocities with $100 \backslash \%, 90 \backslash \%$ and $110 \backslash \%$ of the actual $P$-wave velocity used in the inversion, respectively. $f) \$ \backslash: \backslash \operatorname{sim} \backslash: \$ j)$ are simulated data (horizontal component) using the corresponding S-wave velocities in a)\$: $\backslash$ sim $\backslash: \$ \mathrm{e}$ ) and their dispersion spectra are shown in $\mathrm{k}) \$ \backslash: \backslash \operatorname{sim} \backslash: \$ 0)$, respectively.

$$
261 \times 202 \mathrm{~mm}(300 \times 300 \text { DPI) }
$$




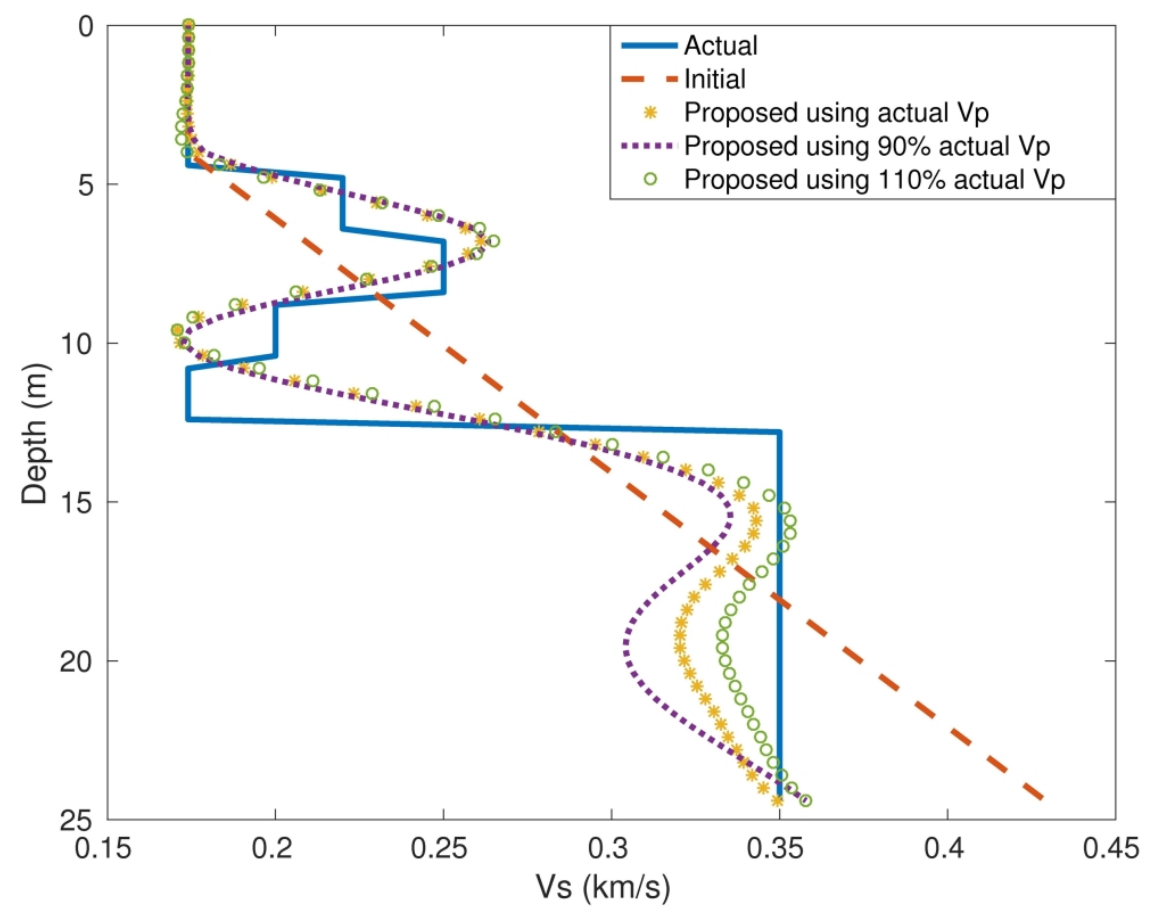

Figure 4 The vertical profile in the middle of the model. The low S-wave velocity zones are captured. The inaccurate $\mathrm{P}$-wave velocities have little influence on the inverted S-wave velocity.

$$
244 \times 183 \mathrm{~mm}(300 \times 300 \mathrm{DPI})
$$


a)

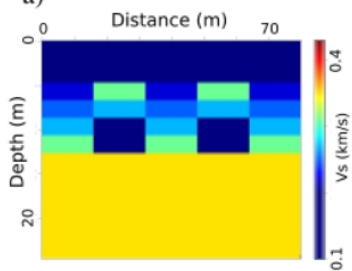

d)

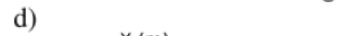

b)

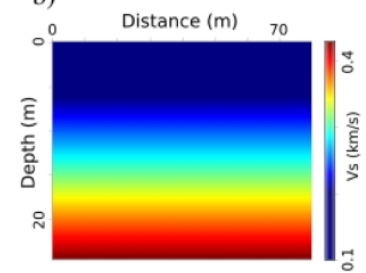

e)
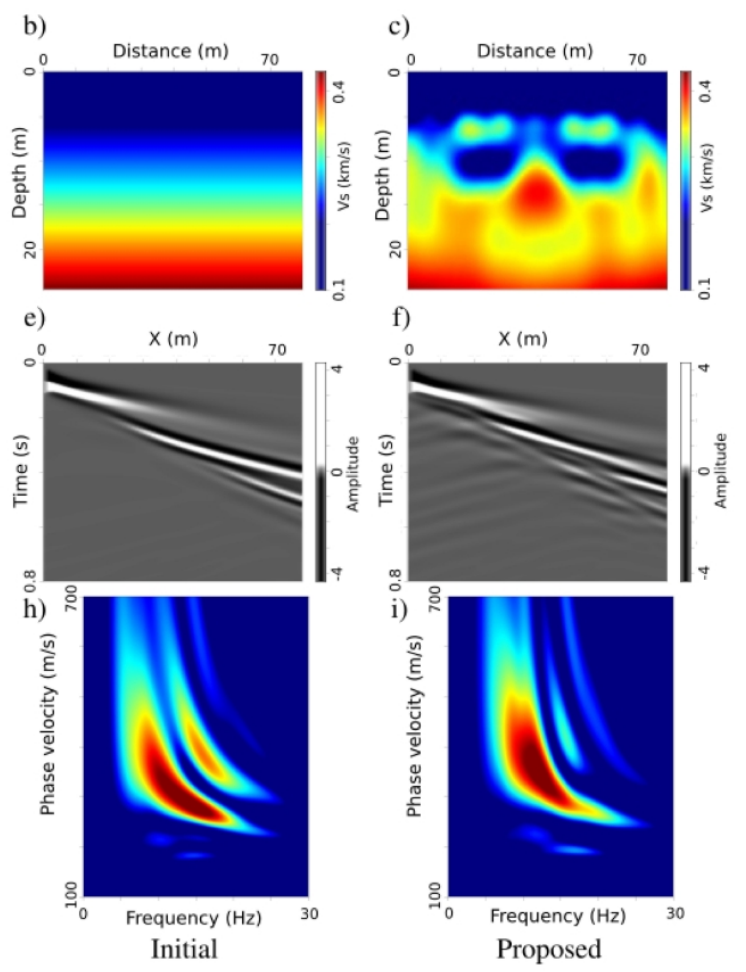

Figure 5 The checkerboard example with a linear gradient initial model. a) Actual checkerboard model, b) linear gradient initial model and c) estimated S-wave velocity. d) $\$ \backslash: \backslash \operatorname{sim} \backslash: \$ f)$ are simulated shot gathers using the S-wave velocities in $\mathrm{a}) \$ \backslash: \backslash \operatorname{sim} \backslash: \$ \mathrm{c})$ and $\mathrm{g}) \$ \backslash: \backslash \operatorname{sim} \backslash: \$ \mathrm{i})$ are corresponding dispersion spectra. The inverted dispersion spectrum is close to the observed one.

$261 \times 202 \mathrm{~mm}(300 \times 300 \mathrm{DPI})$ 

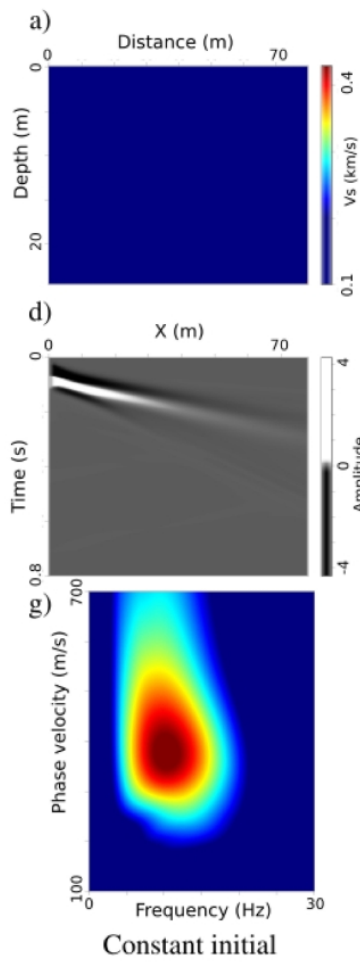

b)

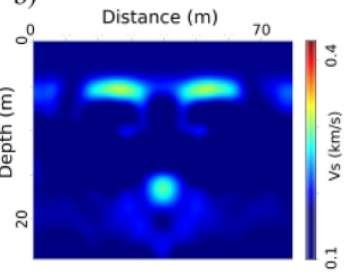

e)

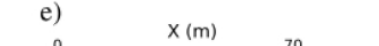

c)

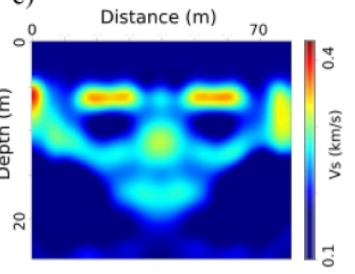

f)

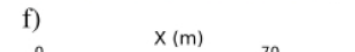

Figure 6 The checkerboard example with a constant initial model. a) The constant S-wave velocity model used for inversion, b) estimated S-wave velocity from conventional elastic FWI and c) the one from our proposed method. The simulated shot gathers are shown in $d) \$ \backslash: \backslash \operatorname{sim} \backslash: \$ f)$ and dispersion spectra of the initial and proposed estimation are shown in $\mathrm{g}$ ) and $\mathrm{i}$ ), respectively. $\mathrm{h}$ ) shows dispersion spectrum obtained from the $\$ \mathrm{~L} \_2 \$$ inversion. The dispersion spectrum of the proposed method is closer to the actual one than the one obtained from $\$ \mathrm{~L} \_2 \$$ inversion.

$$
261 \times 202 \mathrm{~mm}(300 \times 300 \text { DPI })
$$



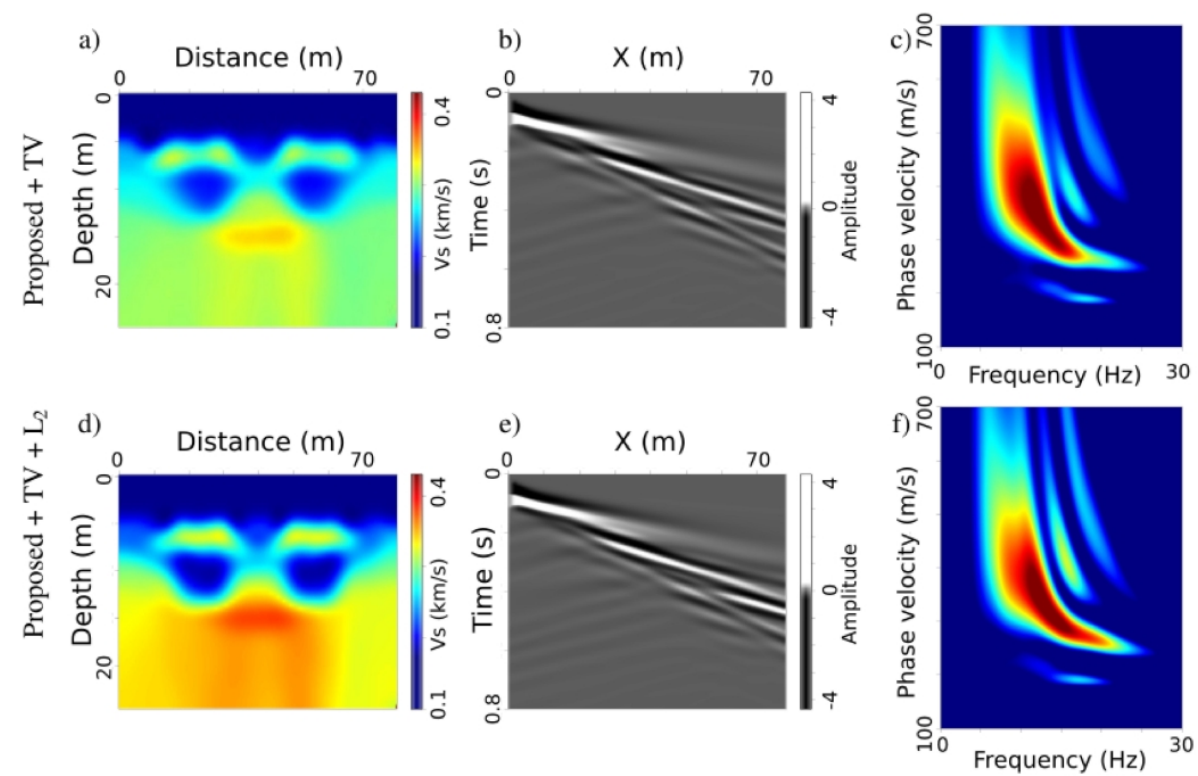

Figure 7 The improved inversion by adding TV constraints to the proposed inversion (Figure $\backslash r$ ref $\{$ fig6 $\} \mathrm{c}$ ). a) $\$ \backslash: \backslash \operatorname{sim} \backslash: \$ c)$ are proposed inversion plus TV constraint. Quality of the deep layer is improved. d) $\$ \backslash: \backslash \operatorname{sim} \backslash:$ f) are conventional elastic FWI results with a) as input model. It indicates that the proposed method plus TV constraint can provide a good initial model for conventional FWI.

$$
261 \times 202 \mathrm{~mm}(300 \times 300 \mathrm{DPI})
$$



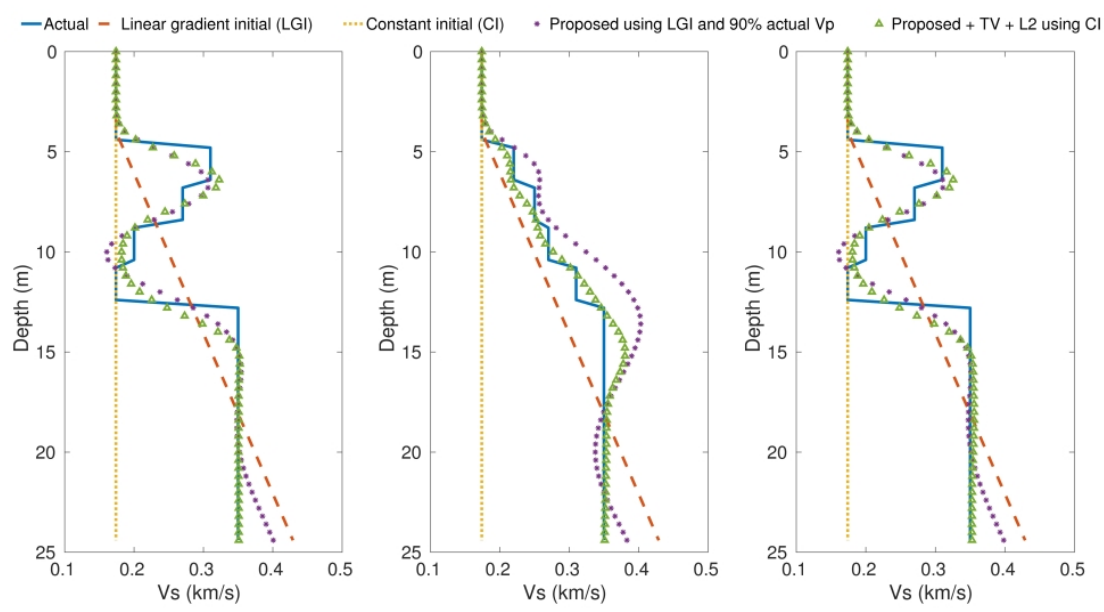

Figure 8 Vertical profiles at different lateral locations (yellow triangles in Figure $\backslash$ ref $\{$ fig5 5 ). There are two pairs of velocities which are 1) linear gradient initial and the proposed inversion and 2) constant initial and $\$ \mathrm{~L} \_2 \$$ norm elastic FWI using the model of proposed inversion plus TV constraints as initial.

$507 \times 240 \mathrm{~mm}(300 \times 300$ DPI) 


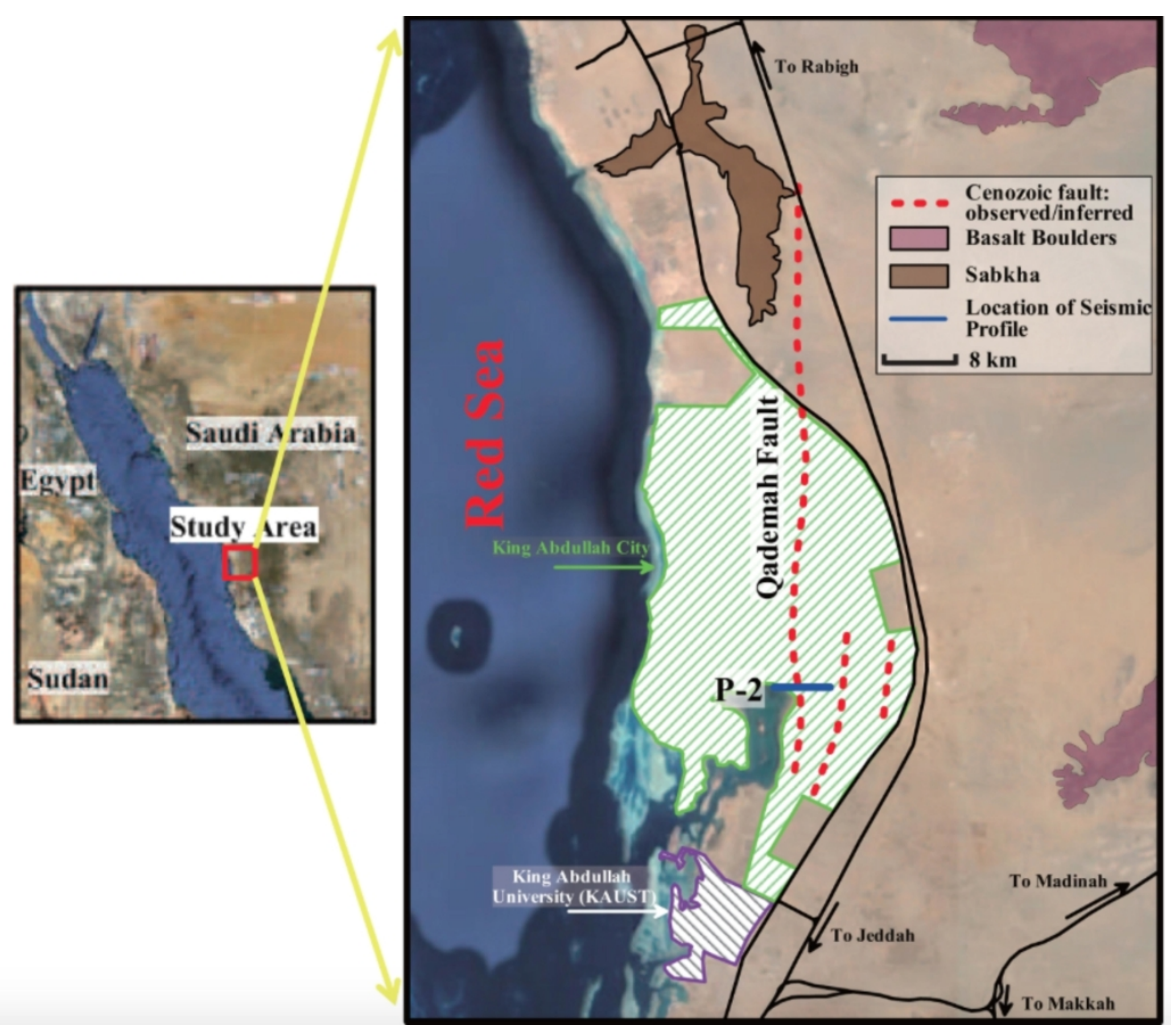

Figure 9 The seismic survey line across the fault area (Hanafy et al., 2015).

$273 \times 211 \mathrm{~mm}(300 \times 300 \mathrm{DPI})$ 
a)

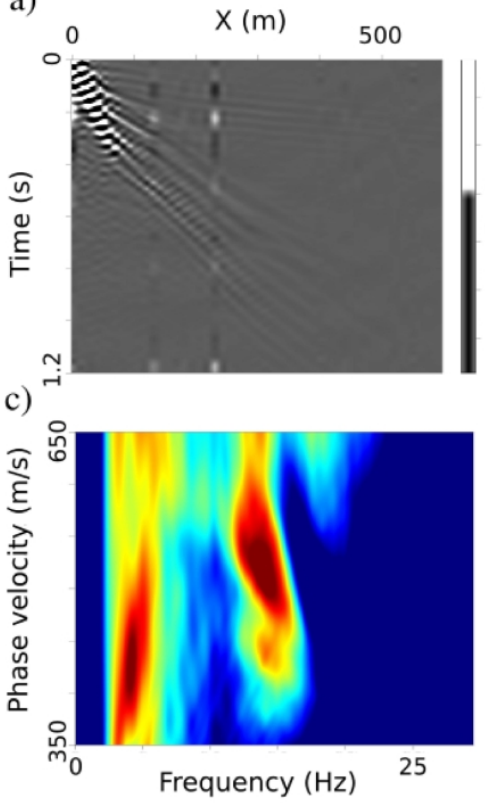

Raw data b)
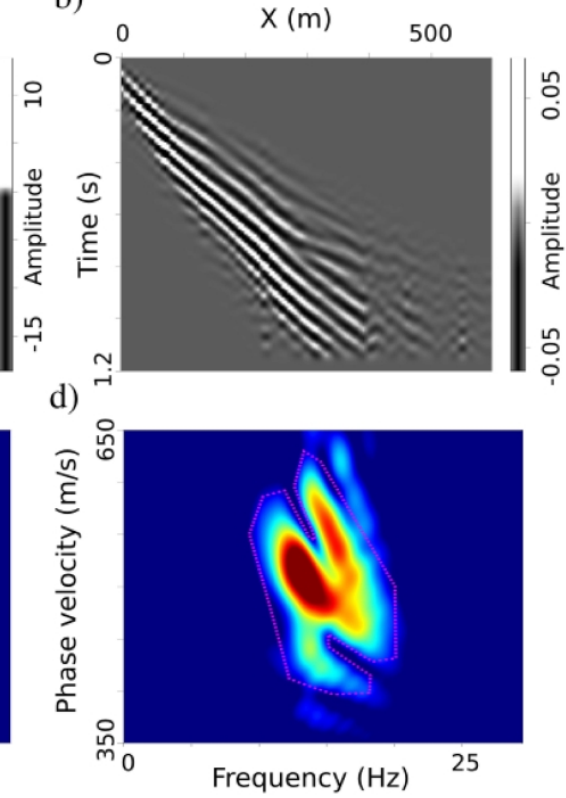

Preprocessed data

Figure 10 Common shot gathers and their dispersion spectra. a) One example raw shot gather and b) preprocessed one. c) and d) are dispersion spectra from a) and b), respectively. The pink contour isolate the dominant modes of Rayleigh waves.

$$
261 \times 202 \mathrm{~mm}(300 \times 300 \text { DPI })
$$




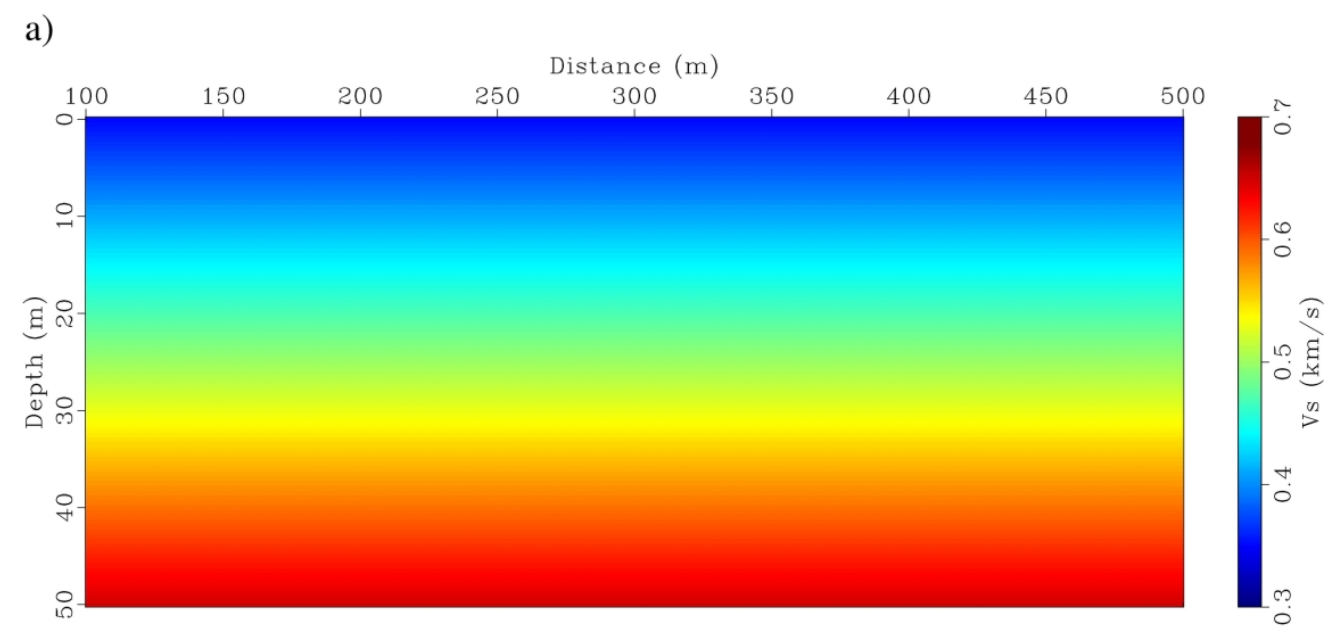

b)

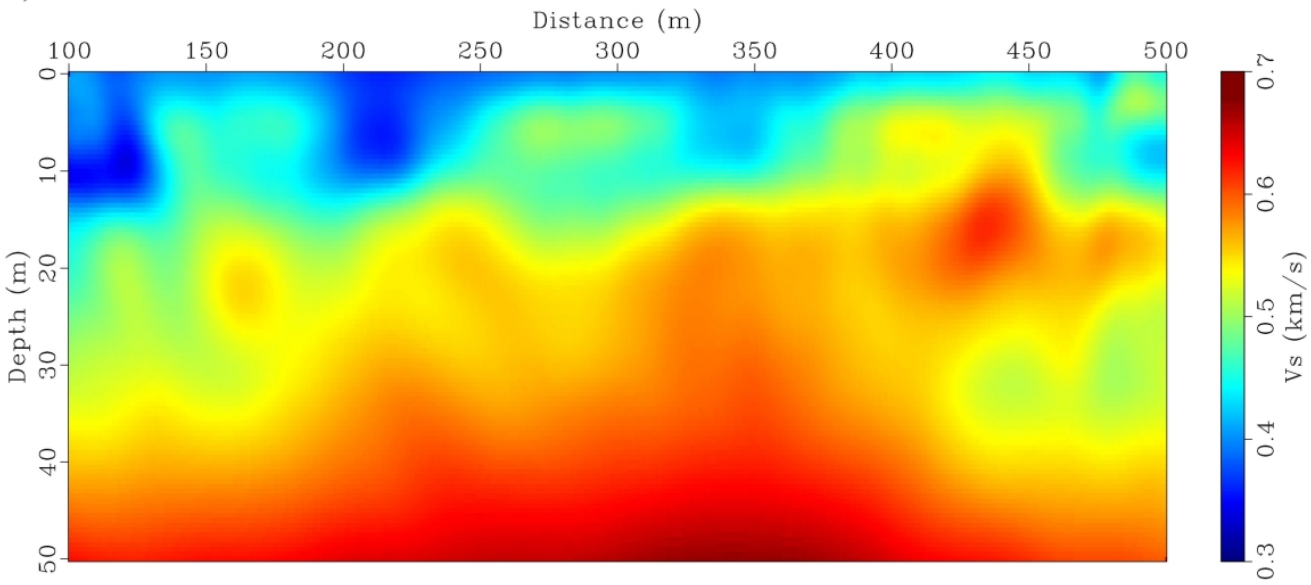

Figure 11 The initial S-wave velocity a) and the estimated one b). The initial S-wave velocity can be roughly determined using the range of velocities in the f-v spectrum (Figure 10d).

$209 \times 198 \mathrm{~mm}(300 \times 300 \mathrm{DPI})$ 


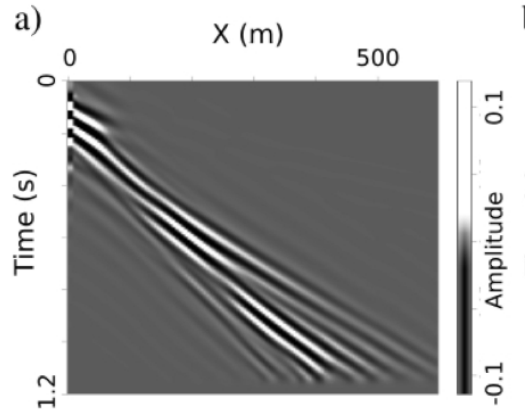

b) $\quad x(m)$

c)

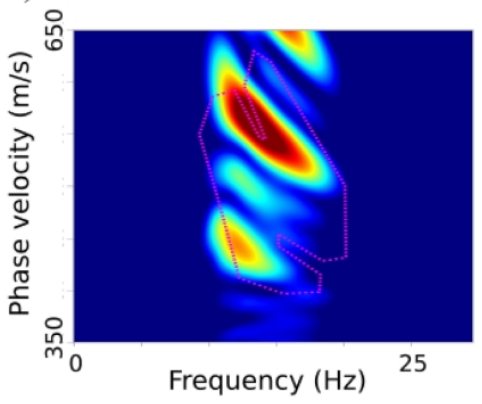

Initial

d)
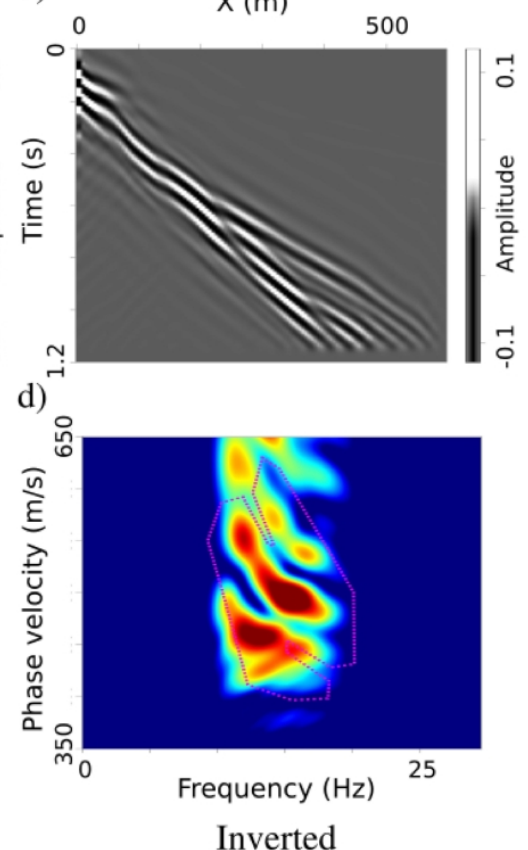

Figure 12 Data comparison. a) and b) are simulated shot gathers using the initial and inverted velocities. c) and d) are the corresponding dispersion spectra. The pink contour indicates the area of dominant modes existing in the observed data.

$261 \times 202 \mathrm{~mm}(300 \times 300$ DPI $)$ 
DATA AND MATERIALS AVAILABILITY

Data associated with this research are available and can be obtained by contacting the corresponding author. 